\title{
Multiscale KF Algorithm for Strong Fractional Noise Interference Suppression in Discrete-Time UWB Systems
}

\author{
Liyun Su, ${ }^{1}$ Yuli Zhang, ${ }^{1}$ Yanju Ma, ${ }^{1}$ Jiaojun $\mathrm{Li}{ }^{2}$ and Fenglan $\mathrm{Li}^{3}$ \\ ${ }^{1}$ School of Mathematics and Statistics, Chongqing University of Technology, Chongqing 400054, China \\ ${ }^{2}$ School of Electronic Information and Automation, Chongqing University of Technology, \\ Chongqing 400054, China \\ ${ }^{3}$ Institute of Library, Chongqing University of Technology, Chongqing 400054, China \\ Correspondence should be addressed to Liyun Su, cloudhopping@163.com
}

Received 26 July 2011; Accepted 4 September 2011

Academic Editor: Daniele Fournier-Prunaret

Copyright (C) 2011 Liyun Su et al. This is an open access article distributed under the Creative Commons Attribution License, which permits unrestricted use, distribution, and reproduction in any medium, provided the original work is properly cited.

In order to suppress the interference of the strong fractional noise signal in discrete-time ultrawideband (UWB) systems, this paper presents a new UWB multi-scale Kalman filter (KF) algorithm for the interference suppression. This approach solves the problem of the narrowband interference (NBI) as nonstationary fractional signal in UWB communication, which does not need to estimate any channel parameter. In this paper, the received sampled signal is transformed through multiscale wavelet to obtain a state transition equation and an observation equation based on the stationarity theory of wavelet coefficients in time domain. Then through the Kalman filter method, fractional signal of arbitrary scale is easily figured out. Finally, fractional noise interference is subtracted from the received signal. Performance analysis and computer simulations reveal that this algorithm is effective to reduce the strong fractional noise when the sampling rate is low.

\section{Introduction}

Ultrawideband (UWB) radio is an emerging technology for future short-rang high-speed wireless communications. In recent years, the Federal Communication Commission (FCC) confined the working bandwidth of UWB systems in the $3.1-10.6 \mathrm{GHz}$ frequency band rang and the power spectral density (PSD) in less than $-41.3 \mathrm{dBm} / \mathrm{MHz}[1,2]$. UWB signal is defined as any wireless signal that has a relative bandwidth more than $25 \%$ or an absolute bandwidth in excess of $500 \mathrm{MHz}$, satisfying PSD required by FCC. Compared with the existing radio communication technologies, the spread spectrum technique of UWB systems 
bears many advantages, for instance, low power consumption, low probability of intercept, strong ability of penetrating materials, and large space capacity.

Since a conventional UWB system transfers data by using ultrashort pulses, it possesses considerably large bandwidths. Usually, it causes mutual interference with the most existing electronic narrowband systems over the same frequency band inevitably, such as radio navigation or detection system, wireless local area network and global positioning system (GPS) [3]. Therefore, to guarantee the successful deployment of UWB, it is related to not only the deployment of efficient multiple access technologies but also narrow-band interference suppression technologies.

Recently a number of the narrowband interference (NBI) suppression technologies have been published thoroughly in many references. Some NBI cancellation solutions [4-11] have been utilized in UWB systems, for example, pulse waveform designing technology, minimum mean square error (MMSE) rake receiver technology, template waveform estimation technology, frequency domain technology, notch filter techniques, and so forth. These methods all selected simple and stable model as NBI model $[12,13]$ and used a large number of estimated channel parameters to eliminate interference. Moreover, they studied less about the nonstationary narrowband interference model and the reduction of channel parameter estimation. Hence, this paper proposes a new multi-scale Kalman filter algorithm for strong fractional noise interference suppression in time hopping (TH) UWB systems. As the nonstationary fractional signal is very strong and UWB signal mixed with white noise is very weak, the received signal in this paper is modeled as narrowband signal which concentrated mainly fractional signal energy. Then it performs the multi-scale wavelet analysis of the received signal and establishes the state space model of Kalman filter estimation [14-17]. The state space model includes a time domain state equation and observation equation. They are obtained by the stationarity of fractional wavelet coefficients. Lastly, we can estimate the fractional Brownian motion (FBM), and a novel multiscale Kalman filter for UWB systems is proposed. Its performance analysis and computer simulations are also discussed in Section 5.

This paper is organized as follows. In Section 2, the discrete-time model of the received signal is defined. In Section 3, we introduce the FBM. A novel multi-scale Kalman filter algorithm is proposed in Section 4. The performance of this multi-scale Kalman filter is analyzed, and simulation results are given in Section 5. The conclusions are drawn in Section 6.

\section{Discrete-Time UWB System Model}

Consider a multiple-access TH-UWB system with single user. The transmitted UWB signal employing binary pulse amplitude modulation (2-PAM) is provided by

$$
s_{t}(t)=\sum_{j=-\infty}^{+\infty} b_{\left\lfloor j / N_{s}\right\rfloor} \omega\left(t-j T_{f}-C_{j} T_{c}\right)
$$

where $\omega(t)$ is a single link transmitted pulse waveform. $T_{f}$ is the pulse repetition period. $T_{c}$ is the TH unit time. $N_{s}$ is the number of repeated pulses over one symbol period. $b_{\left\lfloor j / N_{s}\right\rfloor} \in$ $\{+1,-1\}$ represents the user's information during the $j$ th frame. If a symbol period duration is $T_{b}=N_{s} T_{f}$, the transmitted rate is $R_{s}=1 /\left(N_{s} T_{f}\right) .\left\{C_{j}\right\}$ is the pseudorandom TH sequence which adds an additional time shift $T_{c}$ to each pulse, and $0 \leq C_{j} \leq N_{h}-1$. 
Assume $d_{n}$ stands for the $n$th symbol of single user. Then, (2.1) can be expressed as

$$
s_{t}(t)=\sum_{n=1}^{N_{0}} b_{n} v\left(t-n T_{b}\right)
$$

where $v(t)=\sum_{j=0}^{N_{s}-1} \omega\left(t-j T_{f}-C_{j} T_{c}\right) . N_{0}$ is the number of transmitted symbols.

Assume the nonstationary FBM model is used as NBI model. Then, while the transmitted UWB signal propagates through a flat channel and is corrupted by fractional noise signal $B_{H}(t)$ and additive white Gaussian noise (AWGN) $N(t)$, the observation signal is given by

$$
r(t)=S(t)+B_{H}(t)+N(t)
$$

where $S(t)=\alpha \cdot s_{t}(t) \cdot \alpha$ is the channel gain. $N(t) \sim N\left(0, \sigma_{N_{t}}^{2}\right) . S(t)+N(t)$ denotes the wideband component of the received signal.

However, in practical situation, the received signal must be discrete. Suppose $r(t)$ is sampled at the rate of $1 / T_{s}$ and an output $r(n)=\left.r(t)\right|_{t=n T_{s}}$ is received in discrete-time form. Then, the following received signal becomes

$$
r(n)=B_{H}(t)+S(n)+N(n),
$$

where $S(n)=\alpha \cdot \sum_{m=1}^{N_{0}} b_{n} v(n-m M) . M$ is the number of sampling points in every symbol period.

Thus, in the $n$th symbol period, (2.4) can be changed into

$$
r_{n}=B_{n}+S_{n}+N_{n}
$$

where $S_{n}=X \cdot \eta$ and $X=[v(0), v(1), \ldots, v(M-1)]^{T}$. Here $\eta=\alpha b_{n}$ is a constant.

In this paper, the estimated scheme is proposed to stress against the fractional narrowband interferer. The whole basic idea is to realize the prediction of FBM signal, so that it can be removed from the received signal to reduce the fractional interference. In theory, this paper preforms an analysis of FBM. However, in practice, the estimation of the pure FBM is difficult to achieve because the FBM is often unknown. And as the narrowband signal occupies most of the received signal energy, the prediction of $r(t)$ is used to replace the estimation of FBM. On the other hand, the error signal $r(t)-\widehat{r}(t)$, where $\widehat{r}(t)$ is an estimation of $r(t)$, is very important to eliminate the FBM. Kalman filter technique is also cited to estimate the FBM in this paper. It is shown in Figure 1.

\section{Fractional Brownian Motion}

Brownian motion is a kind of random, irregular motion. In the 1960s, a new theory of fractional Brownian motion, commonly referred to as the FBM, is advanced by Mandelbrot and Van Ness [18].

Denote

$$
B_{H}(t)=\frac{1}{\Gamma(H+1 / 2)} \cdot\left\{\int_{0}^{t}(t-\lambda)^{H-1 / 2} d B(\lambda)+\int_{-\infty}^{0}(t-\lambda)^{H-1 / 2}-(-\lambda)^{H-1 / 2} d B(\lambda)\right\},
$$




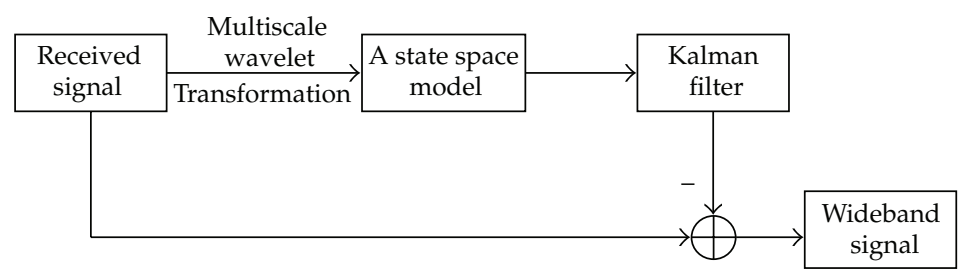

Figure 1: Estimated scheme of fractional noise suppression in UWB systems.

where $\Gamma(\cdot)$ is Gamma function and $H(0<H<1)$ is a Hurst index. For the general Brownian motion, when $H=1 / 2$, it is a standard Brownian motion. Obviously, FBM, in a continuous Gaussian process with a zero mean, has the nonstationarity, self-similarity, and so forth [18, 19].

Certainly, there are many methods of generating Brownian motion. For example, Weierstrass-Mandelbrot random function method, under certain conditions, emerges FBM through an almost everywhere nondifferentiable continuous function; in fractional Gaussian noise method, it is that the fractional Gaussian noise is represented as the incremental derivative of FBM; in covariance matrix transform method, Gaussian white noise is turned into a sequence with the covariance matrix through the Cholesky decomposition technology to get FBM; wavelet transform method, that is to say, in terms of adjacent times frequency conversion rate, makes Gaussian white noise into FBM by the inverse wavelet transform. However, this paper uses Weierstrass-Mandelbrot random function to obtain FBM.

\section{Multiscale Kalman Filter Algorithm on Fractional Noise Model}

With the purpose of NBI suppression to be successfully realized, we consider the discrete orthogonal wavelet decomposition of $B_{H}(t)$. Then, (3.1) can be expressed in the following form:

$$
B_{H}(t)=\sum_{n=-\infty}^{+\infty} c_{J}[n] \Phi_{J, n}(t)+\sum_{j=-\infty}^{+\infty} \sum_{n=-\infty}^{+\infty} d_{j}[n] \Psi_{j, n}(t)
$$

where $a_{J}[n]=2^{-J / 2} \int_{-\infty}^{\infty} I(t) \Phi\left(2^{-J} t-n\right) d t, d_{j}[n]=2^{-j / 2} \int_{-\infty}^{\infty} I(t) \Phi\left(2^{-j} t-n\right) d t . \Phi_{J, n}(t)=$ $2^{-J / 2} \Phi\left(2^{-J} t-n\right)$ is a scale function of the wavelet transform, and the wavelet function is written by $\Psi_{J, n}(t)=2^{-j / 2} \Psi\left(2^{-j} t-n\right)[20,21]$.

If (2.4) is converted applying the wavelet decomposition theory, we have the following equation:

$$
r_{j}[n]=S_{j}[n]+d_{j}[n]+N_{j}[n]=d_{j}[n]+u_{j}[n]
$$

where $j=1,2, \ldots, J \cdot u_{j}[n]=S_{j}[n]+N_{j}[n]$ has the mean $\mu_{j}=S_{j}[n]$ and variance $\operatorname{Var}\left(u_{j}[n]\right)=$ $\sigma_{u_{i}}^{2} \cdot\left\{d_{j}[n]\right\}$ is stable [22]. Then, due to the stationarity of wavelet coefficients $\left\{d_{j}[n]\right\}$, this $\mathrm{AR}(\mathrm{p})$ model can be found in this study:

$$
d_{j}[m]=\sum_{i=1}^{p} a_{i} d_{j}[m-i]+\varepsilon_{j}[m],
$$


where $\varepsilon_{j}[m]$ with a zero mean and variance $\sigma_{\varepsilon_{i}}^{2}$, independent of $S[m]$ and $B_{H}[m]$, is AWGN. $a_{i}, i=1,2, \ldots, p$ are parameter coefficients.

Define

$$
\begin{gathered}
F_{j}=\left[\begin{array}{cc}
O_{\left(M / 2^{j}-1\right) \times 1} & E_{M / 2^{j}-1} \\
O_{1 \times\left(M / 2^{j}-p\right)} & B_{j}^{T}
\end{array}\right], \\
\left.B_{j}=\left[a_{p}, a_{p-1}, \ldots, a_{1}\right]^{T}, \quad G=\left[O_{1 \times\left(M / 2^{j}-1\right)} 1\right]\right]^{T}, \\
d_{j, m}^{\left(M / 2^{j}\right)}=\left[d_{j}\left[m-\frac{M}{2^{j}}+1\right], \ldots, d_{j}[m-1], d_{j}[m]\right]^{T} .
\end{gathered}
$$

For (4.3), we have

$$
d_{j, m+1}^{\left(M / 2^{j}\right)}=F_{j} d_{j, m}^{\left(M / 2^{j}\right)}+G \varepsilon(m+1)
$$

Equation (4.5) is one-step prediction from $d_{j, m}^{\left(M / 2^{j}\right)}$ to $d_{j, m+1}^{\left(M / 2^{j}\right)}$, so there $M / 2^{j}$ iterative steps of $d_{j, n}$ from $d_{j, n+1}$, namely,

$$
d_{j, n+1}=F_{j}^{M / 2^{j}} d_{j, n}+G_{M / 2^{j}} \varepsilon_{n+1},
$$

where $d_{j, n}=d_{j,(n+1) M / 2^{j}-1^{\prime}}^{\left(M / 2^{j}\right.}$ and

$$
\begin{gathered}
G_{M / 2^{j}}=\left[F_{j}^{\left(M / 2^{j}-1\right)} G, F_{j}^{\left(M / 2^{j}-2\right)} G, \ldots, F_{j} G, G\right] \\
\varepsilon_{n+1}=\left[\varepsilon\left(\frac{(n+1) M}{2^{j}}\right), \ldots, \varepsilon\left(\frac{(n+2) M}{2^{j}}-1\right)\right]^{T} .
\end{gathered}
$$

What is more, to facilitate the study below, we need to transform formula (2.5) into an observation equation. That is,

$$
r_{j, n}=d_{j, n}+S_{j, n}+N_{j, n}
$$

Thus, if (4.6) is seen as a state transition equation in a dynamic space, combined with (4.8), we can obtain a state space model for $B_{H}(t)$ :

$$
\begin{gathered}
d_{j, n+1}=F_{j}^{M / 2^{j}} d_{j, n}+G_{M / 2^{j}} \varepsilon_{n+1}, \\
r_{j, n}=d_{j, n}+u_{j, n},
\end{gathered}
$$

where

$$
u_{j, n}=S_{j, n}+N_{j, n}
$$

To estimate the state space model (4.9) in the third part, the new Kalman filter algorithm is structured as follows. 


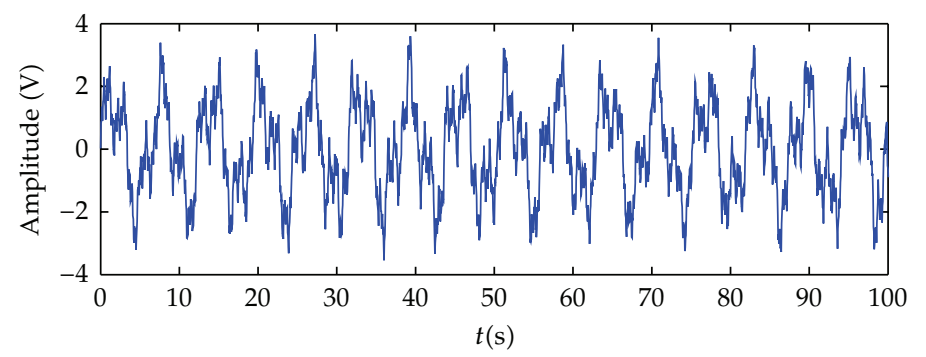

Figure 2: Fractional noise signal generated by Weierstrass-Mandelbrot function.

(1) Initialization: at $n=0$, initialize $d_{j, 0}$ and $P_{0}$ are given.

(2) State update:

$$
\begin{gathered}
\widehat{d}_{j, n+1}^{-}=F_{j}^{M / 2^{j}} \cdot \widehat{d}_{j, n}, \\
P_{j, n+1}^{-}=F_{j, n}^{M / 2^{j}} \cdot P_{j, n} \cdot\left(F_{j, n}^{M / 2^{j}}\right)^{T}+\sigma_{\varepsilon_{j}}^{2} \cdot G_{M / 2^{j}}\left(G_{M / 2^{j}}\right)^{T}, \\
K_{j, n+1}=P_{j, n+1}^{-}\left\{P_{j, n+1}^{-}+\sigma_{u_{j}}^{2}(n) E_{\left(M / 2^{j}\right) \times\left(M / 2^{j}\right)}\right\}^{\dagger}, \\
\widehat{d}_{j, n+1}=\widehat{d}_{j, n+1}^{-}+K_{n+1}\left(r_{j, n+1}-\widehat{d}_{j, n+1}^{-}\right), \\
P_{j, n+1}=\left(I-K_{j, n+1}\right) P_{j, n+1}^{-} .
\end{gathered}
$$

Therefore, this algorithm can predict all the high-frequency coefficients of the wavelet on any scale according to the KF iterative algorithm. It means that FBM can be calculated through the inverse wavelet transform to receive the interference suppression in UWB systems.

\section{Simulation}

In this section, the performance of our proposed NBI suppression method is surveyed. It is simulated by the Matlab7.1 software, and the pulse waveform $\omega(t)$ from the TH-UWB system is adopted as follows:

$$
\omega(t)=\left(1-4 \pi\left(\frac{t}{\tau}\right)^{2}\right) \cdot \exp \left(-2 \pi\left(\frac{t}{\tau}\right)^{2}\right)
$$

where $\tau$ denotes the pulse forming factor. Suppose the parameters are $T_{f}=8 \mathrm{~ns}, N_{s}=2$, $N_{h}=5, M=160$, and $J=3$, and the sampled time interval is $0.05 \mathrm{~ns}$, which is low. Moreover, the Hausdorff-Besicovitch (fractional) dimension of the graph of Weierstrass-Mandelbrot random function is $D=1.5$. Therefore, there are some measured results for this simulation in the following portion. In Figure 2, the original fractional noise signal is shown. Figures 3 and 4 describe the estimated fractional signal and the noisy error signal, respectively. That is, the proposed new algorithm can effectively estimate the strong fractional noise from these results of Figures 1-3. 


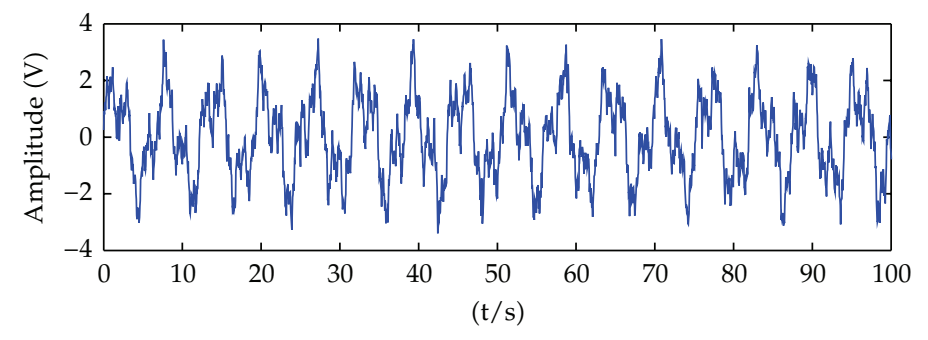

Figure 3: The estimated fractional signal based on multiscale wavelet transform.

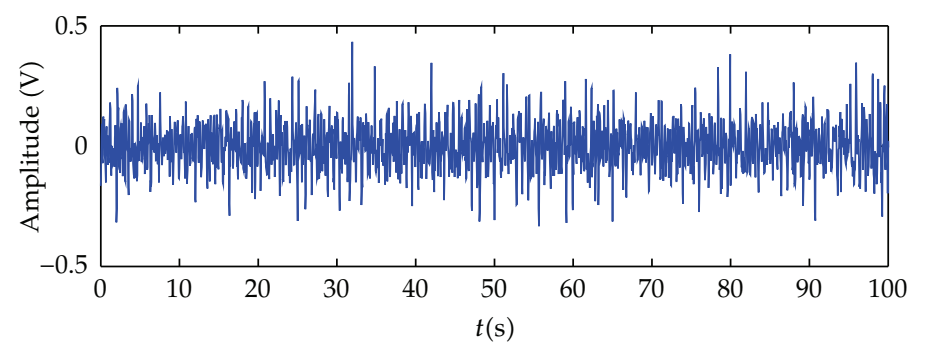

Figure 4: Error signal being wideband component of the observation signal.

Generally, the signal-to-interference ratio (SINR) can detect the performance of a algorithm very well. In order to preferably evaluate the performance of the new fractional noise suppression algorithm from another angle, SINR is improved in this paper:

$$
\begin{aligned}
\mathrm{SINR}_{\text {improvement }} & =\frac{\text { SINR }_{\text {out }}}{\operatorname{SINR}_{\text {in }}}=\frac{E\left\{|r(n)-S(n)|^{2}\right\}}{E\left\{|e(n)-S(n)|^{2}\right\}} \\
& =\frac{\operatorname{Var}\left(B_{H}(n)\right)+\sigma_{N}^{2}}{\operatorname{Var}(e(n))+\sigma_{N}^{2}},
\end{aligned}
$$

where $e(n)=B_{H}(n)-\widehat{B}_{H}(n)$, which is the estimated error signal of $B_{H}(n) . \sigma_{N}^{2}$ is the variance of the white noise.

Thus, 100 independent tests are done, where the lower sampling rate is $0.1 \mathrm{~ns}$ and $D=1.2$ is employed. From this simulation, it is indicated that the actual SINR has greatly improved in Figure 5. On the other hand, it is shown that this new method is effective in suppressing the nonstationary $B_{H}(t)$ for UWB systems, which is a strong NBI.

\section{Conclusion}

In this paper, the multi-scale KF solution is suggested to suppress the fractional noise interference in discrete-time UWB systems. Particularly, after a discrete-time received signal is modeled in the receiver and the fractional signal is transformed based on the wavelet analysis, the fractional signal can be estimated by Kalman filter, which is supposed as a strong nonstationary NBI process. From the simulation above, the algorithm estimates the fractional noise signal under the weak UWB signals by combining the theory of the multi-scale wavelet transform and the Kalman filter. So that it achieves the separation of the narrowband signal 


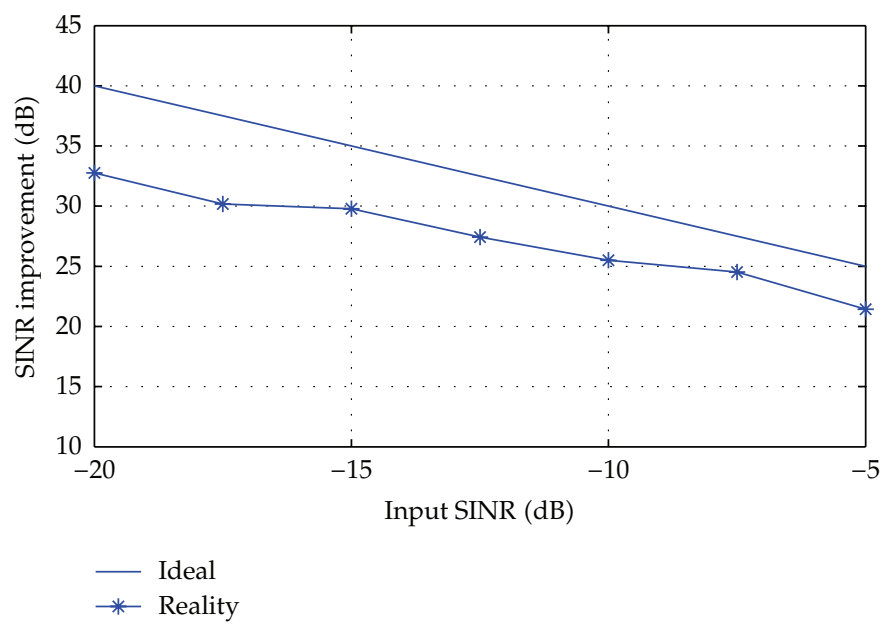

Figure 5: Comparison of the performance of the ideal and proposed multi-scale KF method.

and wideband signals successfully. One of the advantages of this paper is not estimating any channel parameter. Simulation results show that this method is an effective narrowband interference suppression method of UWB systems.

\section{Acknowledgments}

This work was supported by Natural Science Foundation Projects of CQ CSTC of China (CSTC2010BB2310), and Chongqing CMEC Foundations of China (KJ080614, KJ100810, KJ100818).

\section{References}

[1] L. Y. Su and W. Pang, "Ultra-wideband wireless technology-the forefront technology of short-rang wireless communication," Technology and Market, no. 5, pp. 35-36, 2005.

[2] Z. Z. Zhang, X. J. Sha, and Q. Y. Zhang, Ultra-Wideband Communication Systems, Electronic Industry Press, 2010.

[3] G. Zhu, Ultra-Wideband (UWB) Principles and Interference, Tsinghua University Press, 2009.

[4] N. Boubaker and K. B. Letaief, "A low complexity MMSE-RAKE receiver in a realistic UWB channel and in the presence of NBI," in Proceedings of the IEEE Wireless Communications and Networking Conference (WCNC'09), vol. 1, no. 3, pp. 233-237, 2009.

[5] B. Parr, B. L. Cho, K. Wallace, and Z. Ding, "A novel ultra-wideband pulse design algorithm," IEEE Communications Letters, vol. 7, no. 5, pp. 219-221, 2003.

[6] T. Ikegami and K. Ohno, "An interference mitigation study for coexistence of DS-UWB and OFDM," in Proceedings of the 18th Annual IEEE International Symposium on Personal, Indoor and Mobile Radio Communications, (PIMRC'07), vol. 1, no. 9, pp. 583-587, September 2007.

[7] J. Z. Wang and T. T. Wong, "Narrowband interference suppression in time-hopping impulse radio ultra-wideband communications," IEEE Transactions on Communications, vol. 54, no. 6, pp. 1057-1067, 2006.

[8] A. E. C. Tan and K. Rambabu, "Modeling the effects of interference suppression filters on ultrawideband pulses," IEEE Transactions on Microwave Theory and Techniques, vol. 59, no. 1, pp. 93-98, 2010.

[9] K. Ohno and T. Ikegami, "Interference mitigation study for UWB radio using template waveform processing," IEEE Transactions on Microwave Theory and Techniques, vol. 54, no. 4, pp. 1782-1792, 2006. 
[10] R. D. Weaver, "Frequency domain processing of ultra-wideband signals," in Proceedings of the 37th Asilomar Conference on Signals, Systems and Computers, vol. 2, no. 11, pp. 1221-1224, Pacific. Grove, Calif, USA, November 2003.

[11] Y. Dhibi and T. Kaiser, "Impulsive noise in UWB systems and its suppression," Mobile Networks and Applications, vol. 11, no. 4, pp. 441-449, 2006.

[12] X. Chu and R. D. Murch, "The effect of NBI on UWB time-hopping systems," IEEE Transactions on Wireless Communications, vol. 3, no. 5, pp. 1431-1436, 2004.

[13] Y. H. Zhu, Study of Narrowband Interference Suppression in UWB Systems, Yangzhou University, Jiangsu, China, 2009.

[14] M. S. Grewal and A. P. Andrews, Kalman Filtering: Theory and Practice Using MATLAB, John Wiley \& Sons, New York, NY, USA, 2nd edition, 2008.

[15] J. Peng and L. Y. Su, "Performance analysis of blind multi-user detector with fuzzy Kalman filter in IR-UWB," International Journal of Distributed Sensor Networks, vol. 5, no. 1, p. 47, 2009.

[16] Z. F. Ye, Statistical Signal Processing, University Press of Science and Technology, 2008.

[17] M. A. Badamchizadeh, I. Hassanzadeh, and M. A. Fallah, "Extended and unscented Kalman filtering applied to a flexible-joint robot with jerk estimation," Discrete Dynamics in Nature and Society, Article ID 482972, 14 pages, 2010.

[18] B. B. Mandelbrot and J. W. Van Ness, "Fractional Brownian motions, fractional noises and applications," SIAM Review, vol. 10, pp. 422-437, 1968.

[19] X. J. Li, D. X. Nie, and L. G. Yuan, "Computer research with fractional brown motion," Fujian Computer, no. 1, pp. 1-2, 2007.

[20] L. Y. Su, H. Ma, and S. F. Tang, “Weak signal detection of strong fractional noise using chaos oscillator and kalman filtering," Journal of Sichuan University (Engineering Science Edition), vol. 39, no. 3, pp. 149-154, 2007.

[21] L. Su and F. Li, "Deconvolution of defocused image with multivariate local polynomial regression and iterative wiener filtering in DWT domain," Mathematical Problems in Engineering, vol. 2010, Article ID 605241, 14 pages, 2010.

[22] P. Flandrin, "Wavelet analysis and synthesis of fractional Brownian motion," Institute of Electrical and Electronics Engineers. Transactions on Information Theory, vol. 38, no. 2, pp. 910-917, 1992. 


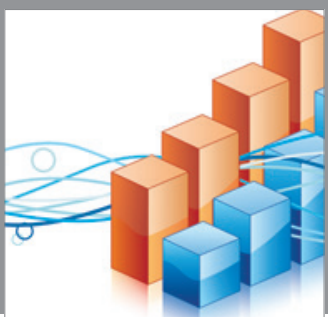

Advances in

Operations Research

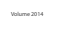

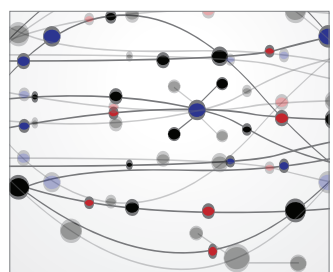

\section{The Scientific} World Journal
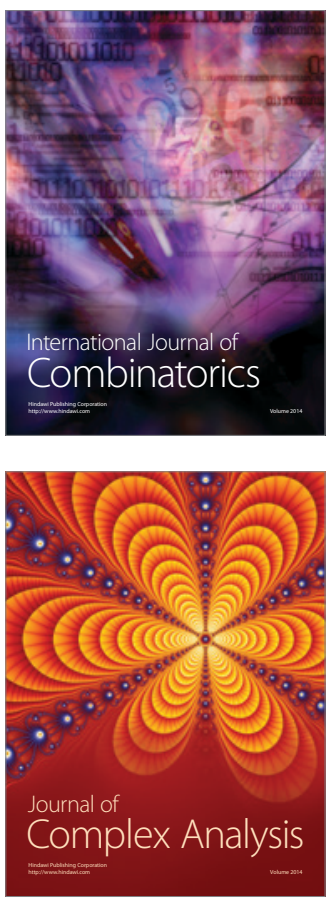

International Journal of

Mathematics and

Mathematical

Sciences
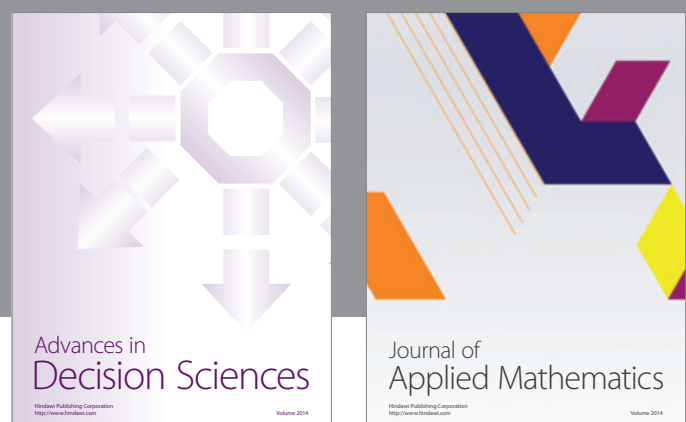

Journal of

Applied Mathematics
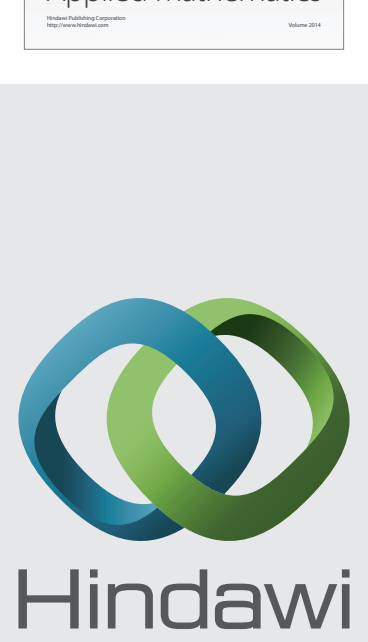

Submit your manuscripts at http://www.hindawi.com
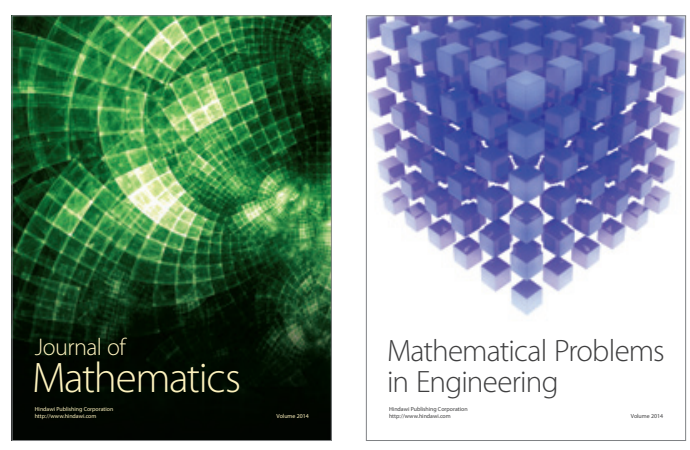

Mathematical Problems in Engineering
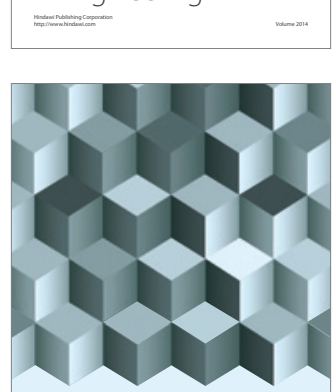

Journal of

Function Spaces
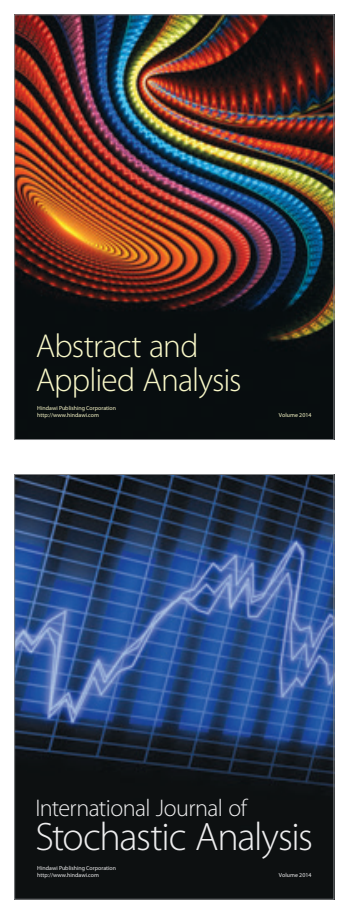

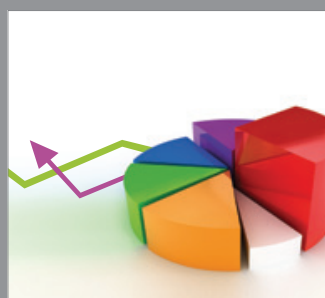

ournal of

Probability and Statistics

Promensencen
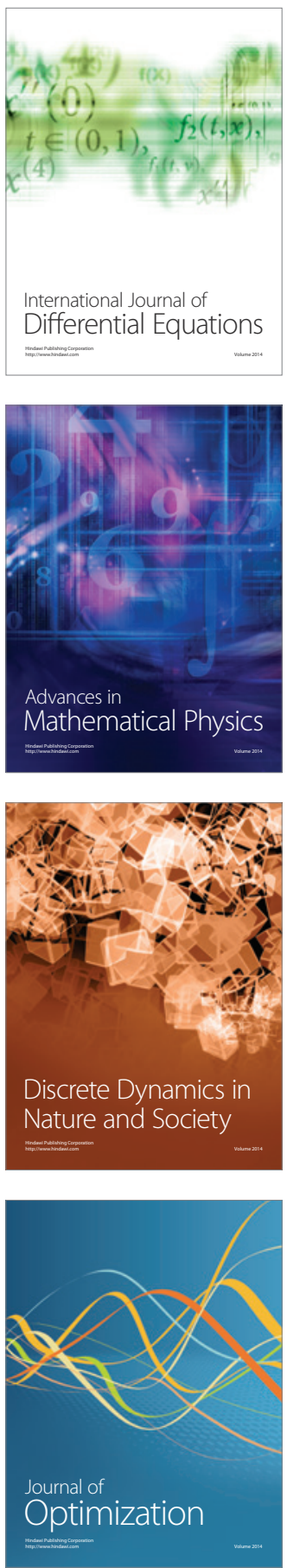